\title{
Internet of things-based vital sign monitoring system
}

\author{
Alamsyah $^{1}$, Mery Subito ${ }^{2}$, Mohammad Ikhlayel ${ }^{3}$, Eko Setijadi ${ }^{4}$ \\ ${ }^{1,2}$ Department of Electrical Engineering, Universitas Tadulako, Indonesia \\ ${ }^{3}$ Department of Information Technology and Communications, Al-Quds Open University, Palestine \\ ${ }^{4}$ Department of Electrical Engineering, Institut Teknologi Sepuluh Nopember, Indonesia
}

\begin{tabular}{l} 
Article Info \\
\hline Article history: \\
Received Nov 10, 2019 \\
Revised May 9, 2020 \\
Accepted May 22, 2020 \\
\hline
\end{tabular}

Keywords:

Health monitoring

Internet of things

Raspberry pi

Sensor

Vital sign

\begin{abstract}
Wireless network technology-based internet of things (IoT) has increased significantly and exciting to study, especially vital sign monitoring (body temperature, heart rate, and blood pressure). Vital sign monitoring is crucial to carry out to strengthen medical diagnoses and the continuity of patient health. Vital sign monitoring conducted by medical personnel to diagnose the patient's health condition is still manual. Medical staff must visit patients in each room, and the equipment used is still cable-based. Vital sign examination like this is certainly not practical because it requires a long time in the process of diagnosis. The proposed vital sign monitoring system design aims to assist medical personnel in diagnosing the patient's illness. Vital sign monitoring system uses HRM-2511E sensor for heart detection, DS18b20 sensor for body temperature detection, and MPX5050DP sensor for blood pressure detection. Vital sign data processing uses a raspberry pi as a data delivery media-based internet of things (IoT). Based on the results of the vital sign data retrieval shows that the tool designed functioning correctly. The accuracy of the proposed device for body temperature is $99.51 \%$, heart rate is $97.90 \%$, and blood pressure is $97.69 \%$.
\end{abstract}

Copyright $@ 2020$ Institute of Advanced Engineering and Science. All rights reserved.

\section{Corresponding Author:}

Alamsyah,

Department of Electrical Engineering,

Universitas Tadulako,

Soekarno Hatta Street, Km. 9 Tondo, Palu, 94118, Indonesia

Email: alamsyah.zakaria@untad.ac.id, alamsyah14@mhs.ee.its.ac.id

\section{INTRODUCTION}

Wireless networks technology-based internet of things (IoT) [1-3] has increased significantly $[4,5]$ and interesting to study, especially smart health services $[6,7]$ such as monitoring systems for blood pressure, heartbeat, and body temperature. IoT-based medical applications and health checks will provide benefits for medical personnel in reducing costs, improving quality of life, strengthening medical diagnoses [8], and subsequent treatment processes [9]. One form of health service that is of particular concern is the examination of vital signs [10]. Based on information from the World Health Organization (WHO), it stated that hypertension [11] and heart disease are the highest health risks. Furthermore, to support health services can be implemented using e-health applications [12]. In 2014 Southeast Asia, especially Indonesia, the death rate reached 35\% due to heart disease, and 39\% experienced age under 44 years. One of the factors causing high mortality in heart disease is slow handling in examining the patient's disease condition [13].

Monitoring vital signs [14] is a top priority that must be considered by health care centers to make early prevention [15] and reduce mortality. Vital sign monitoring used by medical personnel to carry out vital sign patients checks has available at the hospital. However, this equipment still based on conventional [16], which certainly has weaknesses in terms of time efficiency. This condition positively affects patient health services in real-time [17], especially in the process of diagnosing disease, operational costs, and the workload 
of medical staff to increase. IoT-based vital sign monitoring becomes a solution to improve health services in real-time [18] and overcome the problems of conventional medical equipment.

Several researchers are proposing research related to vital signs in diagnosing a patient's disease. Implementation of the proposed design includes heartbeat monitoring [19] and body temperature using Arduino [20, 21] based IoT. Research conducted shows that the heartbeat diagnosis information could control through smartphones [22] and computer server [23, 24]. However, the sensors used by researchers are still limited to heart rate and body temperature sensors. The author has not added a blood pressure sensor in diagnosing a patient's illness. The next research proposed an ability analysis of the routing protocols in health monitoring. The study results show that the recommended temperature heterogeneity energy (THE) method can improve lifetime performance and throughput [25] in the IoT application. For conditions, the number of patients that denser recommended using the optimized link state routing (OLSR) [26, 27] on MANET. However, the researcher has not yet implemented the concept of routing protocols on IoT devices. Patient health monitoring systems are based on simulations to determine IoT performance. The proposed IoT-based vital signs monitoring system expected to reduce the workload of medical personnel and help speed up the process of diagnosing a patient's illness in real-time.

\section{RESEARCH METHOD}

This research using method includes the development of research concepts based on study literature, system design, hardware and software design, hardware testing, software testing, data collection, and analysis of testing results. The research stages of the IoT-based vital sign monitoring system which proposed shown in Figure 1.

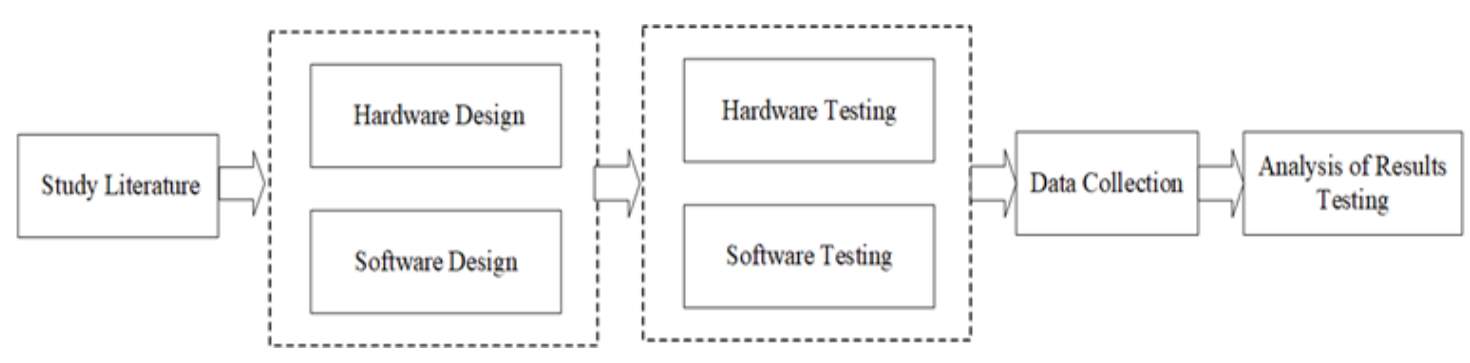

Figure 1. Research methodology

\subsection{System design}

Vital signs measurement using Raspberry Pi is the primary device and data processing. The heart rate sensor as an input reader that provides analog input that is transmitted to Raspberry Pi through the MCP 3008 in converting analog signals to digital so that they can read. Raspberry Pi will process body temperature and blood pressure as digital data input. The results of the retrieval of vital sign data will show on the liquid crystal display (LCD) and Android devices. Furthermore, the results of vital sign checks will be stored in a web server database and can reaccess through the android interface. Figure 2 shows the design of an IoT-based vital sign monitoring system.
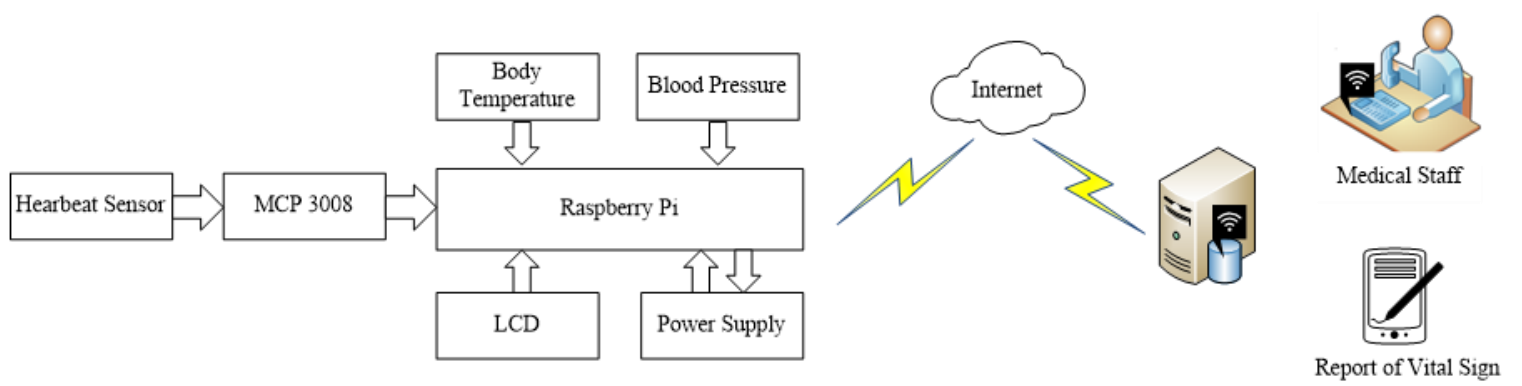

Figure 2. Framework of system design 


\subsection{Hardware design}

Hardware design consists of a series of components, including Raspberry Pi, MCP3008, HRM-2511E sensor, DS18b20 sensor, MPX5050DP sensor, power supply, and LCD. Figure 3 shows that three sensors used as vital sign parameters, namely the HRM-2511E, DS18b20, and MPX5050DP sensors. HRM-2511E sensor serves to detect heart rates in units of beats per minute (BPM). This sensor placed at the tip of the finger, which will produce a heartbeat in the blood circulation as input for analog data for further processing by Raspberry. DS18b20 sensor is a temperature sensor the has accuracy and stability much better than the LM35DZ temperature sensor. This sensor can use to detect body temperature in units of degrees Celsius. MPX5050DP sensor functions to detect blood pressure. Furthermore, vital sign sensor data processed by Raspberry Pi will send to the server and displayed on an android device.

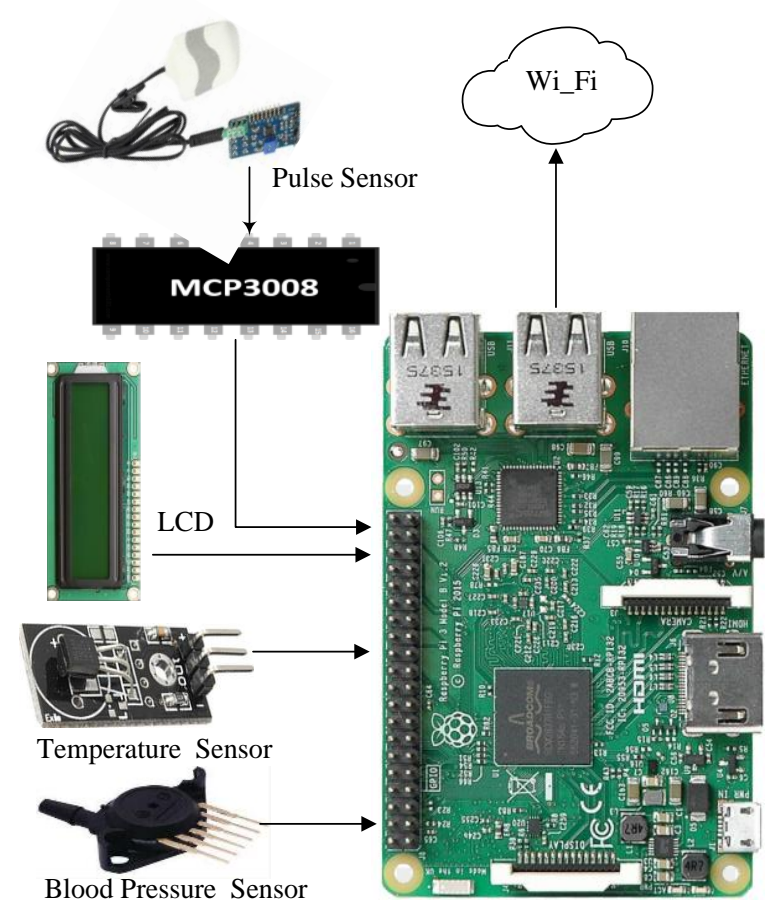

Figure 3. Hardware design

\subsection{Software design}

Raspberry Pi is the main module in the design of processing software. This module functions to program hardware using the Python programming language and stored in a .py extension file. The database system uses MySQL, PHP, and Apache. Figure 4 demonstrates the stages of software.

\subsection{Testing of vital signs device}

Step of next is to test the vital sign sensor, which starts from the preparation of the measuring instrument and verifying the device. This test aims to ensure the sensor is working correctly. First, heart rate sensor testing done by placing one of fingertip onto the sensor. After that, it observed whether the sensor was able to record heart rate data and display the beats per minute (BPM) value. The selection of the fingers and the placement of the fingertips on the pulse sensor will produce more consistent and stable data. Second, the DS18b20 sensor tested with grip the tip of the sensor and observing whether the sensor can display the temperature in units of degrees Celsius. Temperature test results will display on the LCD in degrees Celsius. This test as the basis that the DS18b20 sensor is functioning correctly or not functioning. Body temperature data will vary according to the physical condition of the patient when retrieval data. Third, blood pressure sensor testing done by installing a sensor on the wrist and observing whether the sensor has responded to sensor data by displaying systolic and diastolic values on the LCD. The systolic value is the highest pressure achieved when the heart muscle contracts. The diastolic value is the blood pressure in the arteries when the heart is at rest. 


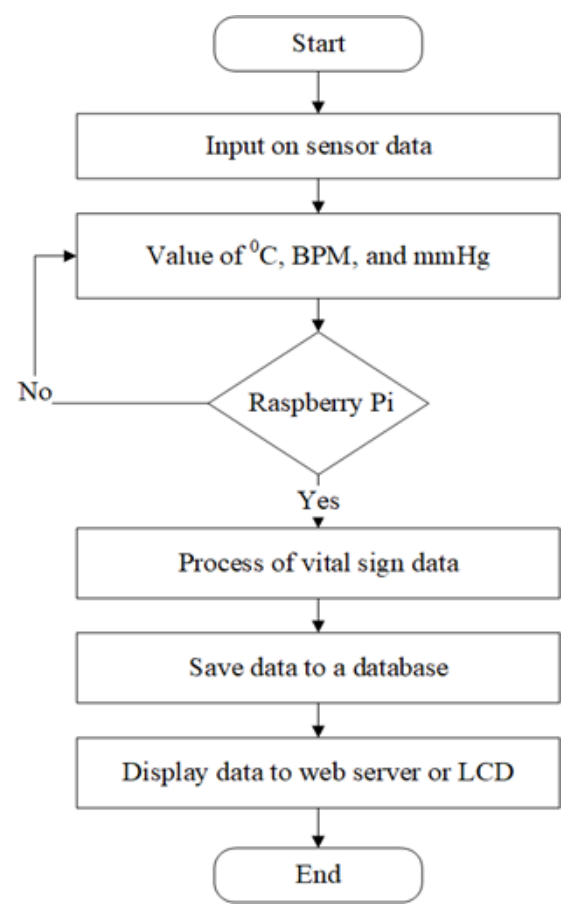

Figure 4. Flow diagram of software

\section{RESULTS AND DISCUSSION}

\subsection{Heartbeat}

Heartbeat data measured to diagnose a patient's heart defects that can cause a heart attack. Heart rate measurements of ten respondents were carried out under normal conditions or not doing activities. To find out how much accuracy and system error in finding the value of the heartbeat. The proposed system design compared with digital pulse sensors sold in the market. Measurements made three times to ensure the accuracy of the resulting data with a time duration of one minute. The data obtained can see in the vital sign database. The measurement of heart rate from a digital device will note on the data collection sheet, which analyzed to conclude all data that has collected. The results of taking IoT-based heartbeat data are in the numbers $78-95$ BPM with an accuracy rate of $2.10 \%$. The heartbeat data obtained still categorized as normal because it is in the range of 60-100 BPM. These results indicate that the proposed design is functioning normally. The result of heartbeat data taking showed in Table 1.

Table 1. Retrieval of heartbeat data

\begin{tabular}{cccc}
\hline Id_Number & \multicolumn{2}{c}{ Heartbeat (BPM) } & Error (\%) \\
& HRM-2511E & Digital hearbeat & \\
\hline 001 & 92 & 94 & 2.13 \\
002 & 86 & 89 & 3.37 \\
003 & 95 & 97 & 2.06 \\
004 & 90 & 93 & 3.23 \\
005 & 87 & 87 & 0 \\
006 & 78 & 82 & 4.88 \\
007 & 89 & 89 & 0 \\
008 & 89 & 91 & 2.19 \\
009 & 92 & 92 & 0 \\
010 & 91 & 94 & 3.19 \\
& Average error & & 2.10 \\
\hline
\end{tabular}

\subsection{Blood pressure}

Taking blood pressure data very determines the condition of a person's health. The normal blood pressure for adults is $90 / 60$ to $120 / 80 \mathrm{mmHg}$. The numbers of $90 \mathrm{mmHg}$ and $120 \mathrm{mmHg}$ are upper blood pressure that indicate the level of heart contractions that push blood through the arteries to the body (systolic). Table 2 shows the results of taking blood pressure data. 
Values of $60 \mathrm{mmHg}$ and $80 \mathrm{mmHg}$ are low blood pressure that shows the amount of blood pressure in the arteries when the heart rests, such as when sleep or silent (diastolic). Measurement and retrieval of blood pressure sensor data performed on ten respondents. Tests carried out three times to see the level of precision of the proposed device. Data generated in digital form by the Raspberry Pi module. The results of data retrieval obtained from a blood pressure sensor compared with a digital blood pressure device that has prepared. This value will use as a comparison between the proposed system design with commercial digital devices. Based on the results of taking blood pressure data for ten respondents showed that the lowest value of $115 \mathrm{mmHg}$ and the highest value of $135 \mathrm{mmHg}$ with an average error rate of $2.31 \%$.

Table 2. Retrieval of blood pressure data

\begin{tabular}{cccc}
\hline Id_Number & \multicolumn{2}{c}{ Blood pressure $(\mathrm{mmHg})$} & Error $(\%)$ \\
& MPX5050DP & Digital blood pressure & \\
001 & 115 & 120 & 4.17 \\
002 & 120 & 125 & 4 \\
003 & 130 & 129 & 0.78 \\
004 & 138 & 136 & 1.47 \\
005 & 130 & 130 & 0 \\
006 & 125 & 125 & 0 \\
007 & 128 & 130 & 1.54 \\
008 & 128 & 130 & 1.54 \\
009 & 135 & 134 & 0.75 \\
010 & 120 & 120 & 0 \\
& Average error & & 2.31 \\
\hline
\end{tabular}

\subsection{Body temperature}

Retrieval of body temperature data aims to analyze the condition of the body temperature experienced by patients. An increase in body temperature will be directly proportional to the rise in heart rate. Neither does the opposite if the body temperature decreases will be causing the heart rate to decrease. Retrieval of body temperature data from ten respondents within three minutes was 36.40 to $36.900 \mathrm{C}$. Temperature data obtained are still in the healthy category for adults. The proceeds of this data collection show that the proposed design is to work fine with an average accuracy of $0.49 \%$. Table 3 shows the results of taking body temperature data.

Table 3. Retrieval of body temperature data

\begin{tabular}{cccc}
\hline Id_Number & \multicolumn{2}{c}{ Body temperature $\left({ }^{0} \mathrm{C}\right)$} & Error $(\%)$ \\
& DS18b20 & Digital thermometer & \\
\hline 001 & 36.48 & 36.60 & 0.33 \\
002 & 36.80 & 36.80 & 0 \\
003 & 36.90 & 36.60 & 0.82 \\
004 & 36.80 & 36.50 & 0.82 \\
005 & 36.90 & 36.40 & 1.34 \\
006 & 36.60 & 36.60 & 0 \\
007 & 36.80 & 36.40 & 1.09 \\
008 & 36.90 & 36.80 & 0.27 \\
009 & 36.80 & 36.70 & 0.27 \\
010 & 36.40 & 36.40 & 0 \\
& Average error & & 0.49 \\
\hline
\end{tabular}

\subsection{Vital sign monitoring system}

Vital sign monitoring application consists of a login menu, data entry (id_number, name, age, gender, address, and telephone number), patient data report, data retrieval process of body temperature, blood pressure, heartbeat, and data storage. Figures 5 and 6 show the method of processing heartbeat, body temperature, and blood pressure data. Data retrieval do by entering patient data. After entering the data, proceed by inserting one of the fingertips into the HRM-2511E sensor. The MPX5050DP sensor mounted on the wrist and the tip of the DS18b20 sensor held. Next, enter the value of the duration of data retrieval into the time menu. After the duration value input, select the process menu. This menu serves to carry out the process of retrieval sensor data attached to the body of the respondent. Respondent data that has recorded will display on the LCD or web server with BPM (heart rate), mmHg (blood pressure), degrees Celsius (body temperature). Vital sign data obtained can save by selecting the save menu. Furthermore, to display respondent data can do by entering respondent data based on Id_Number. 


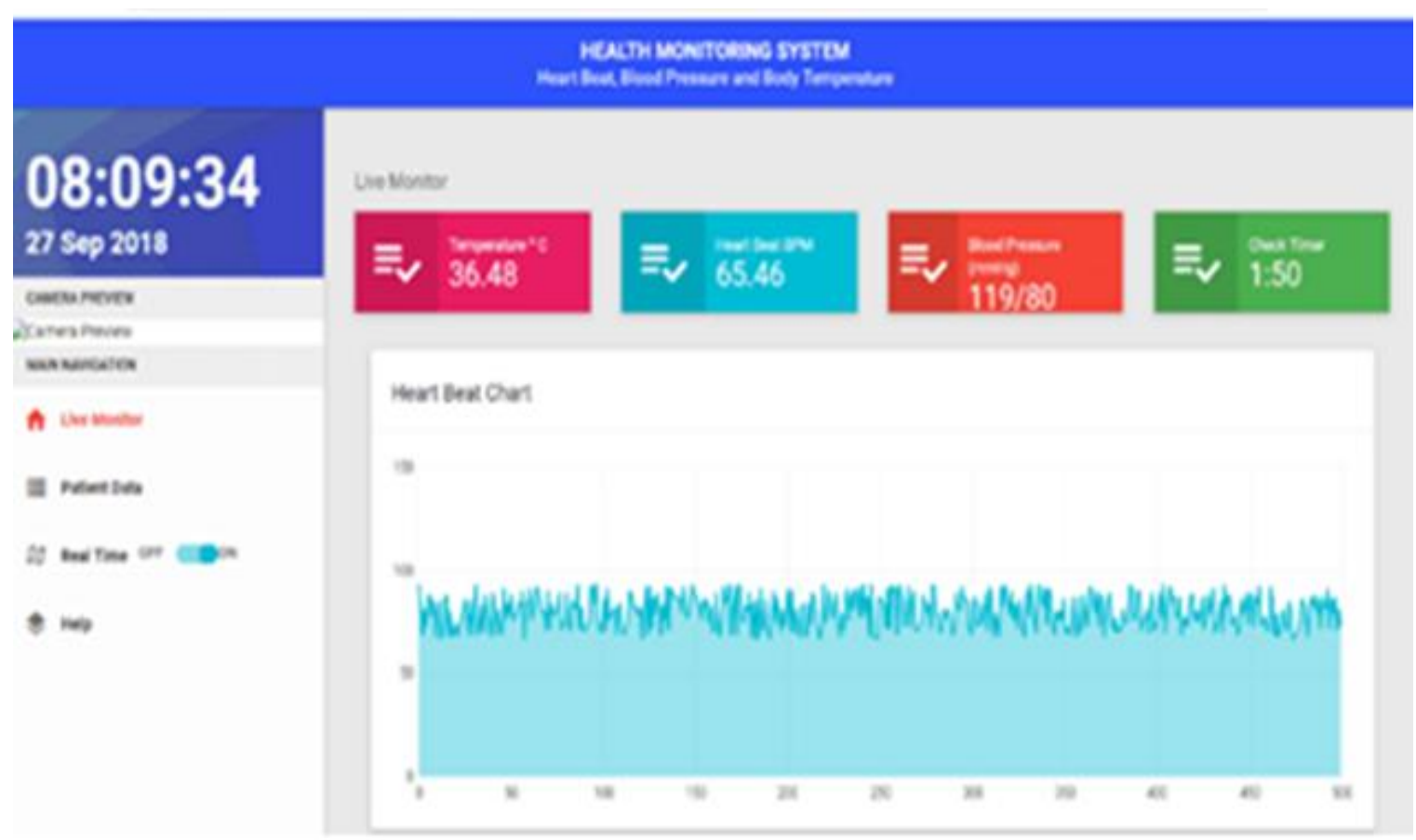

Figure 5. Information of patient's data

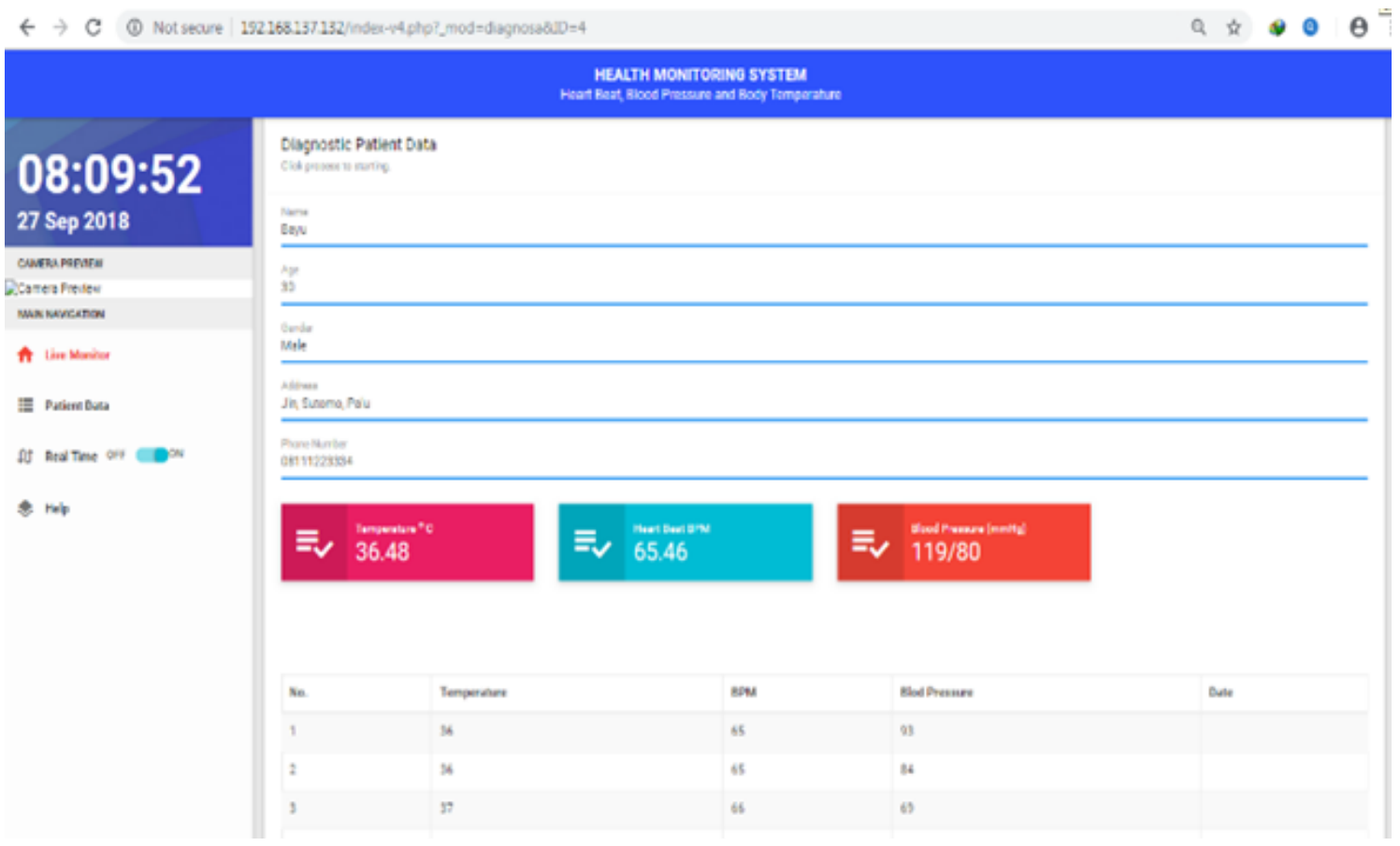

Figure 6. Storage of patient's data

\section{CONCLUSION}

The proposed IoT-based vital signs monitoring system aims to minimize the workload of medic personnel and speed up the diagnosis process of a patient's illness in real-time. Medical staff can access IoT-based vital sign monitoring through an android device. Vital sign data retrieval indicates that the proposed system design is functioning normally. The resulting accuracy level for body temperature is $0.49 \%$, heartbeat of $2.10 \%$, and blood pressure of $2.31 \%$. 


\section{ACKNOWLEDGEMENTS}

Thank you to the Research, Technology, and Higher Education Republic of Indonesia (RISTEKDIKTI) for have to provide funding support Applied Research Grant in 2019 and scholarship support for the Ph.D. program in ITS.

\section{REFERENCES}

[1] D. G. Kristiani, et al., "The Measuring of Vital Signs Using Internet of Things Technology (Heart Rate and Respiration)," IEEE International Seminar on Application for Technology of Information and Communication (iSemantic), pp. 417-422, Sep. 2019.

[2] D. Gupta, et al., "Integrated Healthcare Monitoring Device for Obese Adults Using Internet of Things (IoT)," International Journal of Electrical and Computer Engineering (IJECE), vol. 10, no. 1, pp. 1239-1247, 2020.

[3] N. Nguyen, et al., "A novel autonomous wireless sensor node for IoT applications," TELKOMNIKA Telecommunication, Computing, Electronics and Control, vol. 17, no. 5, pp. 2389-2399, 2019.

[4] Deepika N., et al., "A Novel Three Tier Internet of Things Health Monitoring System," Indonesian Journal of Electrical Engineering and Computer Science (IJEECS), vol. 15, no. 2, pp. 631-637, 2019.

[5] W. Abbass, et al., "Intelligent Risk Management Framework," International Journal of Artificial Intelligence (IJ-AI), vol. 8, no. 3, pp. 278-285, 2019.

[6] N. A. A. Bakar, et al., "The Internet of Things In Healthcare: Anoverview, Challenges and Model Plan for Security Risks Management Process," Indonesian Journal of Electrical Engineering and Computer Science (IJEECS), vol. 15 , no. 1, pp. 414-420, 2019.

[7] V. Sindhura, et al., "An IoT Based Smart Mobile Health Monitoring System", IEEE 2nd International Conference on Inventive Communication and Computational Technologies (ICICCT 2018), pp. 1186-1192, 2018.

[8] N. Ibrahim, et al., "Non-Contact Heart Rate Monitoring Analysis from Various Distances with Different Face Regions," International Journal of Electrical and Computer Engineering (IJECE), vol. 7, no. 6, pp. 3030-3036, 2017.

[9] C. C. Lin, et al., "Wireless Healthcare Service System for Elderly with Dementia," IEEE Transactions on Information Technology in Biomedicine, vol. 10, no, 4, pp. 696-704, Oct. 2006.

[10] Y. Fan, et al., "Vital Sign Measurement in Telemedicine Rehabilitation Based on Intelligent Wearable Medical Devices," IEEE Access, vol. 7, pp. 54819-54823, 2019.

[11] J. Li and Y. Sawanoi, "The History and Innovation of Home Blood Pressure Monitors," IEEE International Conference on Computer Applications \& Information Security (HISTELCON), pp. 82-86, 2017.

[12] WHO, "Global diffusion of eHealth: Making universal health coverage achievable," Global Observatory for eHealth, Dec. 2016.

[13] R. R. Adiputra, et al., "Internet of Things: Low Cost and Wearable SpO2 Device for Health Monitoring," International Journal of Electrical and Computer Engineering (IJECE), vol. 8, no. 2, pp. 939-945, 2018.

[14] K. Bhagchandani and D. P. Augustine., "IoT Based Heart Monitoring and Alerting System with Cloud Computing and Managing the Traffic for an Ambulance in India," International Journal of Electrical and Computer Engineering (IJECE), vol. 9, no. 6, pp. 5068-5074, 2019.

[15] S. Misbahuddin, et al., "Automatic Patients' Vital Sign Monitoring by Single Board Computer (SBC) Based MPI Cluster," IEEE International Conference on Computer Applications \& Information Security (ICCAIS), pp. 1-5, 2019.

[16] M. A. Yusof and Y. W. Hau, "Mini home-based vital sign monitor with android mobile application (Myvitalgear)," IEEE-EMBS Conference on Biomedical Engineering and Sciences (IECBES), pp. 150-155, 2019.

[17] A. Hodge, et al., "Wireless Heart Rate Monitoring and Vigilant System," IEEE International Conference for Convergence in Technology (I2CT), pp. 1-5, 2018.

[18] M. Shu, et al., "The Vital Signs Real-Time Monitoring System Based on Internet of Things," IEEE International Conference on Information Science and Control Engineering (ICISCE), pp. 747-751, 2017.

[19] R. R. Rajanna, et al., "An IoT Wi-Fi Connected Sensor for Real Time Heart Rate Variability Monitoring," IEEE Third International Conference on Circuits, Control, Communication and Computing, pp. 1-4, 2018

[20] N. A. Zakaria, et al., "IoT (Internet of Things) Based Infant Body Temperature Monitoring," International Conference on BioSignal Analysis, Processing and Systems (ICBAPS), pp. 148-153, 2018.

[21] K. Kalaithasan, et al., "Internet of Things Application in Monitoring Sick Building Syndrome," Indonesian Journal of Electrical Engineering and Computer Science (IJEECS), vol. 12, no. 2, pp. 505-512, 2018.

[22] M. Irmansyah, et al., "Low Cost Heart Rate Portable Device for Risk Patients with IoT and Warning System," IEEE International Conference on Applied Information Technology and Innovation (ICAITI), pp. 46-49, 2018.

[23] A. Gutte and R. Vadali, "IoT Based Health Monitoring System Using Raspberry Pi," IEEE International Conference on Computing Communication Control and Automation (ICCUBEA), pp. 1-5, 2018.

[24] T. S. Sollu, et al., "Monitoring System Heartbeat and Body Temperature Using Raspberry Pi," E3S Web of Conferences, vol. 73, pp. 1-5, 2018.

[25] E. Selem, et al., "The (Temperature Heterogeneity Energy) Aware Routing Protocol for IoT Health Application," IEEE Access, vol. 7, pp. 108957-108968, 2019.

[26] Alamsyah, et al., "Performance of the Routing Protocols AODV, DSDV and OLSR in Health Monitoring Using NS3," IEEE International Seminar on Intelligent Technology and Its Application (ISITIA), pp. 323-328, 2016. 
[27] Alamsyah, et al., "Performance Analysis of AODV, AOMDV, and DSDV Routing Protocols in MANET Based Quality of Service," IEEE International Seminar on Application for Technology of Information and Communication. (iSemantic), pp. 286-289, 2018.

\section{BIOGRAPHIES OF AUTHORS}
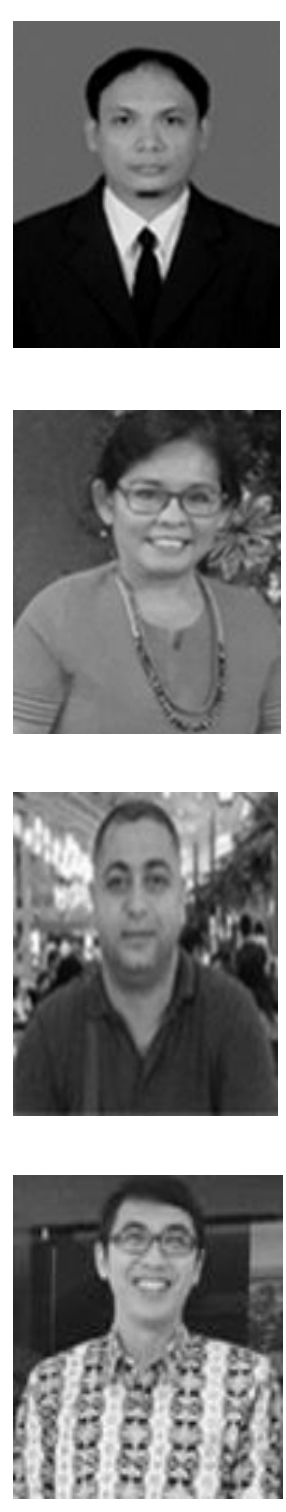

Alamsyah received his bachelor degree in Electrical Engineering Department of Hasanuddin University of Indonesia, Makassar, Indonesia in 2000. He received his Master of Electrical Engineering from Hasanuddin University, Makassar, Indonesia, in 2010. He has joined Department of Electrical Engineering, Tadulako University Palu, Indonesia, and Indonesia Since 2002. His current interest's research areas are Communication Network, WSN, Biomedical Engineering, and Artificial Intelligence. He is currently pursuing the Ph.D. Degree at Department of Electrical Engineering, Institut Teknologi Sepuluh Nopember, Surabaya, Indonesia, since 2014.

Mery Subito is a senior lecturer at the Department of Electrical Engineering, Tadulako University, Indonesia. She earned his Masters of Engineering in the field of electronics engineering at Gajah Mada University, Indonesia in 2002. Her fields of research interest Digital Electronics, Microcontrollers, Telecommunications Basics, and Signal Processing. Her recent research work focuses on the biomedical field specifically the development of vital sign monitoring infrastructure.

Mohammed Ikhlayel is a Faculty member at Al-Quds Open University (QOU). He was Action Head of Department of Information Technology and Communications from 2009 until 2012, in QOU. Mr. Ikhlayel received his Bachelor degree in electrical engineer (Communications) in 2004, he received his master degree in Communications in 2007 from Egypt, and he Studies $\mathrm{PhD}$ at Department of Electrical Engineering, Institut Teknologi Sepuluh Nopember, Surabaya, Indonesia, since 2016.

Eko Setijadi received his bachelor degree in electrical engineering from Institut Teknologi Sepuluh Nopember (ITS), Surabaya, Indonesia in 1999. He received his Master of Technology from Institut Teknologi Bandung, Bandung, Indonesia in 2002. He received a Ph.D. degree from Kumamoto University, Japan in 2010. His research interest is in structural health monitoring, wireless sensor network, computer network, microwave device, antenna design, multimedia, and electromagnetic computing. 